\title{
Thermospheric Dynamics during the March 1989 Magnetic Storm
}

\author{
William J. Burke \\ Boston College, Chestnut Hill, MA, USA
}

E mail (wjhk.burke@comcast.net).

Accepted: 3 July 2018

\begin{abstract}
During the magnetic superstorm of 13-14 March 1989, approximately 2500 space objects in low Earth orbits (LEO) were "lost" by the U.S. Space Surveillance Network. Modeling disturbance effects on satellite drag intensified throughout the following decades but met with limited success. The launch of the GRACE satellites in 2002 offered new hope for advancing orbit-prediction capabilities under harsh geomagnetic conditions. This paper reviews some recent gains in understanding thermospheric environments based on analyses of GRACE data and an adaptation of the Jacchia [1977] model. We show that exospheric temperatures, and consequent neutral density profiles, can be predicted using either solar wind and interplanetary magnetic field parameters or the Dst index. Here, lessons learned are applied, first to estimate the degree of thermospheric heating during the March 1989 storm then compare results with GRACE measurements during the three largest storms between 2002 and 2008 . The highest mass density increases, by about a factor of two, occurred during the 13-14 March 1989 storm. Our analysis suggests that solar EUV fluxes, parametrized by $\sqrt{F_{10.7}}$, play subtle but critical roles for explaining the unrepeated mass density and satellite drag levels experienced during the March 1989 storm.
\end{abstract}

\section{(c) 2018 BBSCS RN SWS. All rights reserved}

Keywords: Magnetic storms, Thermospheric heating, Satellite drag

\section{Introduction:}

The March 1989 magnetic storm was a water shed for raising awareness of hazardous space weather effects on the operation of both ground and space based systems. Two of the best-known effects are the nine-hour outage of electrical power produced by Hydro Québec and the loss of about 2500 space objects being tracked by the US Space Surveillance Network (SSN). Over the following decade electric companies learned to respond effectively to this space weather threat by improving circuit grounding of power-generating plants. In spite of great efforts, only limited success was achieved in predicting satellite drag during severe geomagnetic storms [Bowman et al., 2008].

The launch of the Gravity Recovery and Climate Experiment (GRACE) satellites in March 2002 opened a new era for specifying stormtime responses of the global thermosphere [Bruinsma et al., 2004, 2006] in which tracked space objects can be lost, thereby degrading collisions avoidance capabilities [Wright, 2007]. GRACE consists of two spacecraft that fly in nearly circular polar orbits, separated by $\sim 220 \mathrm{~km}$. Thermospheric densities $(\rho)$ are derived from the Spatial Triaxial Accelerometers for Research (STAR) sensor that monitor the electrostatic forces needed to maintain a proof mass (PM) at the center of a cage located within $2 \mathrm{~mm}$ of the satellites centers of mass [Bruinsma et al., 2004]. Both the spacecraft and PM respond to gravity-induced changes in the same way. However, atmospheric drag affects the spacecraft, but not the PM. Drag forces are determined from changes in electrostatic forces needed to maintain the PM at the STAR cage's center [Bruinsma and Biancale, 2003].

Burke et al. [2007] compared GRACE measurements of thermospheric densities, sampled once per second, during the magnetic disturbances of 7 - 11 November 2004 with predictions of the Jacchia-77 and MSIS models. Both models used the 3-hour Ap index to mimic sources of geomagnetic disturbances. While model predictions agreed with GRACE mass-density $(\rho)$ measurements during quiet periods, they significantly underestimated variations encountered during disturbed intervals. However, cross polar cap potentials $\left(\Phi_{P C}\right)$, estimated using solar wind and IMF measurements from the Advanced Composition Explorer (ACE) satellite, and the Dst index closely tracked the centroid of $\rho$ variations sampled during disturbances. The fact that both $\Phi_{\mathrm{PC}}$ and Dst can be predicted from the same interplanetary parameters [Burton et al., 1975] suggested that perhaps, in similar fashion, they also influence the global thermosphere's stormtime variability. To test this conjecture, Burke [2008] initiated detailed examinations of: (1) numerical relationships between J77 parameters and (2) with GRACE density measurements. The internal parameter study demonstrated quadratic relations between $\rho$ at any height $h$ and the exospheric temperatures $\left(T_{\infty}\right)$ which control mass density-versus-height profiles. An application of the first law of thermodynamics showed that the globally average exospheric temperature $\bar{T}_{\infty}$ is linearly proportional to the total energy in the thermosphere $\left(E_{t h}\right)$. Results of the initial GRACE-based study of the November 2004 storm suggested that with the spacecraft being at nearly constant altitude it would be useful to use orbit-averaged values of the various parameters based on measured densities. We define orbitaveraged density as $\bar{\rho}=1 / N \sum_{i=1}^{i=N} \rho_{i}$ where $i=1$ and N mark times when a GRACE orbit successively crosses ascending nodes, 92 minutes apart.

Our analysis showed that: (1) exospheric temperatures $\left(T_{\infty}\right)$ control mass density versus height profiles, (2) $T_{\infty}$ strongly correlates with $\bar{\rho}$ at thermospheric heights $(h)$, and (3) orbitaveraged $T_{\infty}$ measured by polar orbiting spacecraft (such as GRACE), are linearly proportion to the total energy of the global thermosphere.

The March 1989 storm was a singular space-weather event in the post-Sputnik era. While the event itself remains poorly understood, its outcome bodes ill for future operations, as nearEarth space becomes more densely populated [Wright, 2007]. What was so special about the space environment prevailing when so many LEO objects were lost? The answer to that question is of vital importance. One can imagine "worst-case scenarios" in which the trajectories of massive "lost" objects are set on paths that intersecting that of the International Space Station. Normally, ground controllers would initiate collision avoidance procedures. But, what might happen if controllers lacked critical information needed to recognize the impending peril? This paper seeks to demonstrate a new method to improve understanding of conditions under which very dangerous satellite drag environments grow, by examining thermospheric characteristics that may have prevailed the 13-14 March 1989 storm period, based on lessons learned 
during our analyses of GRACE data acquired during severe storms of comparable magnitudes.

The following section briefly reviews relevant aspects of the Jacchia models and their adaptation as tools for analyzing GRACE measurements, during the magnetic storms of July and November 2004. This analysis revealed that stormtime thermosphere acts as a driven-dissipative system driven by the solar wind and IMF in ways similar to Dst predictions described by Burton et al. [1975]. Burke [2011] applied this method to analyze almost all magnetic storms sampled by GRACE between 2002 and 2008. It demonstrated that solar EUV fluxes, parametrized by the 81-day averaged $\sqrt{F_{10.7}}$ index, modulate solar wind-to-thermosphere coupling coefficients. The Discussion section has two parts in which we first apply these findings to reconstruct thermospheric responses during the March1989 storm. Calculated results are validated through comparisons with GRACE measurements acquired during the three most intense storms between 2002 and 2008. The second part points out two obvious deficiencies of our global approach and suggests possible paths for addressing them.

\section{Methodology:}

This section has three major subsections. The first reviews relevant aspects of the Jacchia (1977) model. The second subsection applies modeling results to an analysis of GRACE data during storm periods in July and November 2004. The third subsection considers effects of solar-cycle modulations of EUV fluxes affect the intensities of stormtime thermospheric responses.

\section{Jacchia Model and Interplanetary Perspectives:}

The J77 model contains 19 empirically-based tables listing mass densities, compositions and temperatures as functions of altitude $(h)$ and exospheric temperature over the ranges 90 to 2600 $\mathrm{km}$ and $500 \leq T_{\infty} \leq 2600{ }^{0} \mathrm{~K}$, respectively [Jacchia, 1977]. Burke [2008] treated the contents of $\mathbf{J} 77$ tables as real data, and used a least-square polynomial fitting to establish the relationship

$$
T_{\infty}=\sum_{i=1}^{2} a_{i} \rho^{i}(h)
$$

across the altitude range 300 to $500 \mathrm{~km}$. Values of the $a_{i}(h)$ coefficients were established using higher order polynomial fits. Burke [2008] also demonstrated that the total thermospheric energy $\left(\bar{E}_{t h}\right)$ in Joules is linearly related to globally averaged exospheric temperatures $\left(\bar{T}_{\infty}\right)$ as

$$
\bar{E}_{t h}=5.36510^{17}+8.72710^{13} \bar{T}_{\infty}
$$

over the range $700 \leq \mathrm{T}_{\infty} \leq 2000{ }^{0} \mathrm{~K}$. In all cases, fitting procedures applied to $\mathrm{J} 77$ tables yielded correlation coefficients $>0.99$.

To quantify solar connections to geophysical processes, Burke et al. [2009] (cf. Appendix 2) proposed using a Volland-Stern like electric field parameter $\varepsilon_{\mathrm{VS}}=\Phi_{\mathrm{PC}} / 2 L_{Y}$, the cross-polar cap potential divided by the width of the magnetosphere along the dawn-dusk meridian. In units of Earth radii $\left(\mathrm{R}_{\mathrm{E}}\right)$, $L_{Y} \approx 14.4 \sqrt[6]{P_{S W}(n P a)}$, where $P_{S W}$ is the dynamic pressure of the solar wind. Following a suggestion by Hill [1984], Siscoe et al. [2002] argued that $\Phi_{P C}=\frac{\Phi_{I} \Phi_{S}}{\Phi_{i}+\Phi_{S}}$ where the symbols $\Phi_{\mathrm{I}}$ and $\Phi_{\mathrm{S}}$ represent contributions of the solar wind /IMF and a saturation effect, respectively. Earlier linear studies indicated that $\Phi_{I}=\Phi_{0}+L_{G} V_{S W} B_{T} \sin ^{2} \frac{\theta}{2}$. Here $\Phi_{0} \approx 25 \mathrm{kV}$ is a residual potential, probably contributed by the low latitude boundary layer. $L_{G} \approx 3.5 \mathrm{R}_{\mathrm{E}}$ is the width of a gate in the solar wind through which geo-effective equipotentials pass [Burke et al., 1999]; $B_{T}=\sqrt{B_{Y}^{2}+B_{Z}^{2}}$, and $\theta=\cos ^{-1}\left(\mathrm{~B}_{Z} / \mathrm{B}_{\mathrm{T}}\right)$ is the IMF clock angle in the $Y-Z$ plane, respectively. MHD simulations indicate $\Phi_{S}(k V)=\frac{1600 \sqrt[3]{P_{S W}(n P a)}}{\Sigma_{P}(S)} \quad$ [Siscoe et al., 2002]. In a follow-on study of the March 2001 magnetic storm, Ober et al., [2003] confirmed the essential correctness of the Siscoe-Hill model. Best fits to measured $\Phi_{\mathrm{PC}}$ were obtained with polar cap conductivities $\Sigma_{\mathrm{P}} \approx 10$ Siemens. The conclusions of this analysis were confirmed using the same technique in all magnetic storms reported here for which all required ACE data were available. We note however that Boudouridis et al. [2004] identified a case in which $\Phi_{\mathrm{PC}}$ measured by a DMSP satellite exceeded the Siscoe-Hill saturation limit after a pressure pulse in the solar wind passed by Earth. In a subsequent MHD simulation, Ober et al. [2007] reproduced this result and found that such responses are transient and soon returned to the Siscoe-Hill limit.

\section{Early GRACE Data Analyses:}

Burke et al. [2009] focused on the analysis of all GRACE data initially available at the Air Force Research Laboratory They covered the intervals 29 May - 18 August and $1-25$ November 2004. Relevant data, plotted as functions of day of the year, from the first and second intervals appear in the left and right sides of Figure 1, respectively. From top to bottom, the traces provide the

Dst index, hour-averaged $\boldsymbol{\varepsilon}_{\mathrm{vs}}$ derived from ACE measurements, and orbit-averaged of $\rho, T_{\infty}$, and $\mathrm{E}_{\mathrm{th}}$. The bottom two parameters were calculated using GRACE measurements of $\rho$ and the J77 model. Looking first at the left side of Figure 1, we see that traces of $\bar{\rho} \varepsilon_{V S}, T_{\infty}$ and $E_{t h}$ varied on two time scales: (1) low-frequency variations periods $\sim 15$ days, and (2) rapid increases that that correlate with intensifications of Dst and $\boldsymbol{\varepsilon}_{\mathrm{VS}}$. The more intense variables observed between days 204 and 211, plotted on the right side of Figure 1 are discussed below

Attention is now directed to the Dst plot that shows that two major geomagnetic disturbances occurred between days 312 and 315 (7-10 November). Dst minima of $-373 \mathrm{nT}$ and -289 nT occurred at 06:00 UT on 8 November and 10:00 UT on 10 November, respectively. Similar disturbances appear in all other stormtime data plots.

Figure 2 shows $\mathrm{J} 77$ predictions of $\mathrm{Ln} E_{t h}$ and hourly averaged

$\mathcal{E}_{V S}$ from the magnetically disturbed interval 22 - 29 July 2004. Attention is directed to two intervals on days 208 and 210 delineated by pairs of vertical lines. In each case the interval's beginning coincided with a precipitous decrease in the $\boldsymbol{\varepsilon}_{\mathrm{VS}}$ trace towards zero. Note too that during both intervals the slope of the Ln $\delta$ Eth traces were straight lines. This observation indicates that the excess thermospheric thermal energy was decreasing exponentially. The corresponding exponential decay-time coefficient $\tau_{\mathrm{E}}$ is $\sim 6.5$ hours, presumably via black body radiation. This observation suggested that during storms the global thermosphere acts like a large driven-dissipative system. 

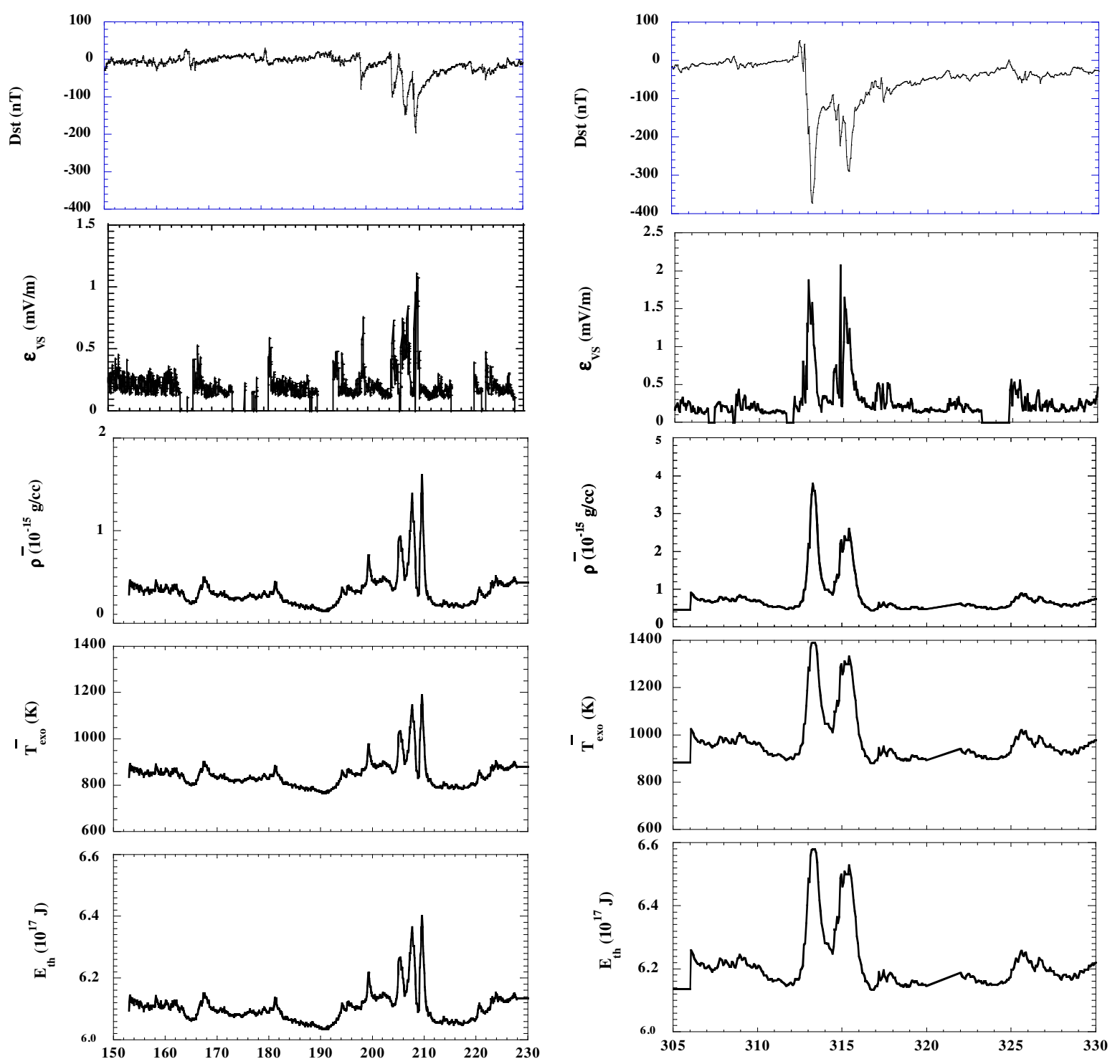

Figure 1. Data acquired between 29 May and 18 August (left) and 1-25 November 2004 (right). From top to bottom, plots show as functions of UT: (1) the Dst index, (2) hourly averaged $\varepsilon_{V s}$, (3) the logarithm of $\bar{\rho}$ in grams per cc, measured by GRACE at altitudes near $495 \mathrm{~km}$, (4) $T_{\infty}$ in degrees Kelvin and (5) $E_{t h}$ in units of $10^{17} \mathrm{~J}$, predicted $\mathrm{J} 77$.

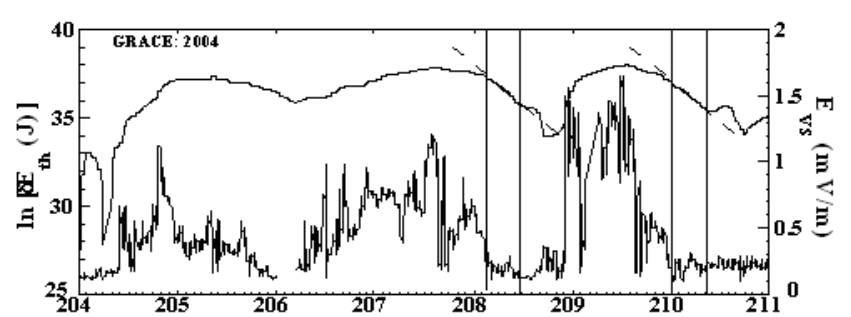

Figure 2. Ln $E_{\text {th }}$ (smoothly varying line) and $\mathcal{E}_{\text {vs }}$ (rapidly varying line) derived from GRACE and ACE measurements, plotted as functions of UT during the $22 \div 29$ July 2004 disturbed period.

Burke et al. [2009] tested this hypothesis by considering the equations that describe driven-dissipative systems for comparison with GRACE measurements,

$$
\frac{d \delta E_{t h}}{d t}=\alpha_{E} \varepsilon_{V S}-\frac{\delta E_{t h}}{\tau_{E}} \quad \text { and } \quad \frac{d \delta T_{\infty}}{d t}=\alpha_{T} \varepsilon_{V S}-\frac{\delta T_{\infty}}{\tau_{T}}
$$

The symbols $\delta E_{t h}$ and $\delta T_{\infty}$ indicate solar-wind driven variations of $E_{t h}$ and $T_{\infty}$ above quiet $E_{Q}$ th and $T_{Q \infty}$, pre-storm values. The coupling coefficients $\alpha_{\mathrm{E}}$ and $\alpha_{\mathrm{T}}$ were determined from values of $E_{t h}$ and $T_{\infty}$ when their time derivatives pass through zero.
During the 2004 storms shown in Figure $1, \alpha_{\mathrm{E}}$ and $\alpha_{\mathrm{T}}$ were about $5.5 \times 10^{15}(\mathrm{~J} / \mathrm{hr}) /(\mathrm{mV} / \mathrm{m})$ and $63\left({ }^{\circ} \mathrm{K} / \mathrm{hr}\right) /(\mathrm{mV} / \mathrm{m})$, respectively; $\tau_{\mathrm{E}}=\tau_{\mathrm{T}} \approx 6.5 \mathrm{hrs}$. With these coefficients, $\delta E_{t h}$ and $\delta T_{\infty}$ traces, obtained with measured $\mathcal{E}_{V S}$ in the driven-dissipative equations, closely approximate those derived using GRACE measurements of $\bar{\rho}$ shown in Figure 1. Burke et al. [2009] also showed that a modified version of the driven-dissipative equation for Dst [Burton et al., 1975],

$$
\frac{d D s t}{d t}=\alpha_{D} \varepsilon_{V S}-\frac{\delta D s t}{\tau}
$$

with $\alpha_{\mathrm{D}}=-40(\mathrm{nT} / \mathrm{hr}) /(\mathrm{mV} / \mathrm{m})$ and $\tau_{\mathrm{D}}=7.7$ hours closely replicated Dst traces in Figure 1. It is important to note that the

two events highlighted in Figure 2 where $\boldsymbol{\varepsilon}_{\mathrm{VS}}$ falls rapidly to near zero during a storm's early recovery phase was quite unusual. Consequently, we used $\tau_{\mathrm{E}}=6.5 \mathrm{hrs}$ in all driven-dissipative calculations

As fuller data sets became available from the GRACE satellites, $T_{\infty}$ was calculated for each $\bar{\rho}$ measurement. Burke [2011] compared $\delta T_{\infty}$ and Dst traces obtained during the 38 magnetic storms, between 2002 and 2008, when $\boldsymbol{\varepsilon}_{\mathrm{VS}}$ 
measurements were available, with predictions of drivendissipative equations (3) and (4), using $\boldsymbol{\alpha}_{\mathrm{T}}$ and $\boldsymbol{\alpha}_{\mathrm{D}}$ cited above. While the temporal shapes of the calculated and measured curves were similar, their amplitudes were not. Rather, $\alpha_{T}$ and $\alpha_{D}$ values that gave best amplitude fits during the 38 storms were second order polynomial functions of 81-day averaged $\sqrt{\left\langle F_{10.7}\right\rangle}$

$$
\begin{aligned}
& \alpha_{T}=-256.7+48.3 \sqrt{F_{10.7}}-1.86 F_{10.7}, \\
& \alpha_{D}=157.4-33.8 \sqrt{F_{10.7}}+1.44 F_{10.7}, \\
& \alpha_{T} / \alpha_{D}=3.88-0.784 \sqrt{F_{10.7}}+0.029 F_{10.7}
\end{aligned}
$$

Over the past two decades, space weather researchers have become used to having ready access to information about interplanetary drivers available from the ACE satellite. This need not be the case. For example, during the 2003 Halloween storm, solar $\mathrm{MeV}$ protons contaminated the low-energy particle sensor on ACE, making it impossible to specify solar wind densities. To make allowance for such cases Burke [2011] investigated the possibility of using the Dst, rather than $\boldsymbol{\varepsilon}_{\mathrm{vs}}$ as a parametric driver for estimating properties of the stormtime thermosphere. Combining equations (3) and (4),

$$
\begin{aligned}
& \frac{1}{\alpha_{T}}\left[\delta T_{\infty}\left(t_{n+1}\right)-\left(1-\frac{1}{\tau_{T}}\right) \delta T_{\infty}\left(t_{n}\right)\right]=\varepsilon_{V S}(t) \\
& \varepsilon_{V S}(t)=\frac{1}{\alpha_{D}}\left[\operatorname{Dst}\left(t_{n+1}\right)-\left(1-\frac{1}{\tau_{D}}\right) \operatorname{Dst}\left(t_{n}\right)\right]
\end{aligned}
$$

and eliminating $\boldsymbol{\varepsilon}_{\mathrm{Vs}}$ gives

$$
\begin{aligned}
\delta T_{\infty}\left(t_{n+1}\right) & =\left(1-\frac{1}{\tau_{T}}\right) \delta T_{\infty}\left(t_{n}\right)+ \\
+ & \frac{\alpha_{T}}{\alpha_{D}}\left[\operatorname{Dst}\left(t_{n+1}\right)-\left(1-\frac{1}{\tau_{D}}\right) \operatorname{Dst}\left(t_{n}\right)\right]
\end{aligned}
$$

To solve equation (7) numerically we set $\delta T_{\infty}\left(t_{0}\right)$ and $\operatorname{Dst}\left(t_{0}\right)$ as 0 ; the $\alpha_{T} / \alpha_{D}$ ratio is specified by the concurrent values of $\left\langle\mathrm{F}_{10.7}\right\rangle$. During the 2003 Halloween storm, the full solar wind data set needed to calculate $\varepsilon_{\mathrm{Vs}}$ were unavailable. This provided an opportunity to test equations (5) and (7) with $F_{10.7}$ and $\alpha_{T} / \alpha_{D}$ were 140 and 1.33, respectively. Figure 6 of Burke [2011] shows that $\delta \mathrm{T}_{\infty}$ curves derived from GRACE data and equation (7) closely approximate one another.

\section{Application to the March 1989 Storm:}

Success in applying equation (7) to the Halloween 2003 magnetic storm suggests that the technique can also be used to calculating the history of $\delta \mathrm{T}_{\infty}$ during the March 1989 storm. However, this is just a first step toward estimating mass density distributions in the stormtime thermosphere. Hourly-averaged mass densities in the global thermosphere require some prior knowledge about the quiet time exospheric temperatures $\left(T_{Q} \infty\right)$ from which the stormtime $\delta T_{\infty}$ departs in the main phase and returns in recovery

Data plotted in Figure 4 shows the relationship $T_{\infty} Q$ and $\left\langle F_{10.7}\right\rangle$ in the red-line fits of Figure 3. The linear statistical analysis (red line) in Figure 4 indicates that $T_{\infty}$ and $\left\langle F_{10.7}\right\rangle$ have a correlation coefficient $\mathrm{R}>0.99$. During the first half of March 1989 , $\left\langle\mathrm{F}_{10.7}>\right.$ was $\sim 208$ sfu. The linear correlation equation predicts that $\mathrm{T}_{\mathrm{Q}} \approx 1135{ }^{\circ} \mathrm{K}$ and $\left\langle F_{10.7}\right\rangle=208$. Equation (5) requires that $\alpha_{T} / \alpha_{\mathrm{D}} \approx-1.4$. Numerically, equation (7) reduces to:

$\delta T_{\infty}\left(t_{n+1}\right)=0.846 \delta T_{\infty}\left(t_{n}\right)-1.4 \operatorname{Dst}\left(t_{n+1}\right)-1.22 \operatorname{Dst}\left(t_{n}\right)$
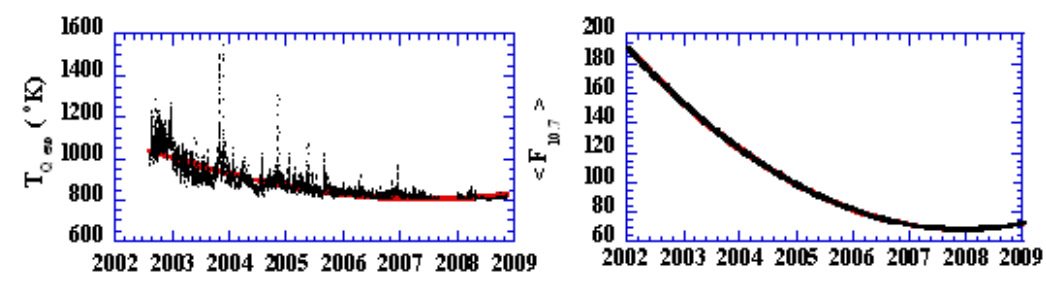

Figure 3. The left plot shows orbit-averaged $T_{\infty}$ inferred from GRACE

measurements of $\bar{\rho}$ between mid-2002 through the end of 2008. The right plot shows 81 -day averaged $\left\langle\mathrm{F}_{10.7}\right\rangle$ over the same interval. Superposed red lines represent $4^{\text {th }}$ order polynomial fits to these data.

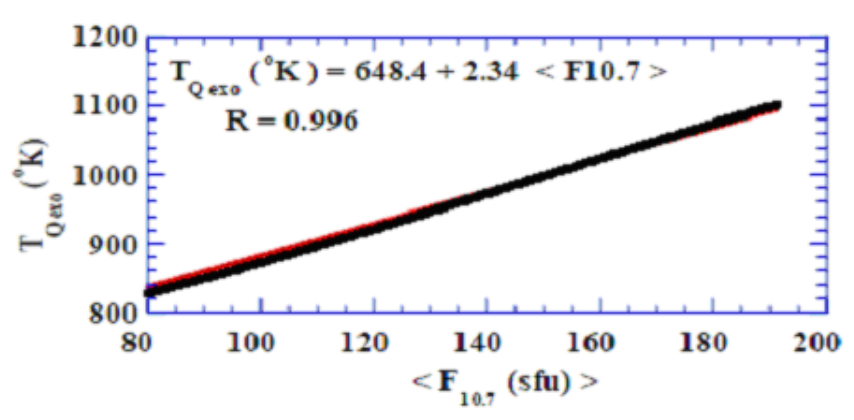

Figure 4. $T_{\infty Q}$ plotted as a function of 81-day averaged $F_{10.7}$ acquired between mid-2002 and the end of 2008. A linear correlation analysis between these variables appears as a red line. The linear regression coefficient is $>0.99$.
The bottom plot of Figure 5 shows that Dst reached a main phase minimum of $-589 \mathrm{nT}$ at 02:00 UT 14 March. On this basis, numerical values of $\delta T_{\infty}\left(\mathrm{t}_{\mathrm{n}+1}\right)$ were calculated subject to initial conditions $\delta T_{\infty}\left(\mathrm{t}_{0}\right)$ and Dst $\left(t_{0}\right)=0$. The calculated maximum $\delta T_{\infty} \approx 652{ }^{\circ} \mathrm{K}$ was reached near 01:00 UT on 14 March. The middle panel of Figure 5 shows the $T_{\infty}=T_{\infty} Q+\delta T_{\infty}$ across the two day period. Its highest calculated value was $T_{\infty} \approx 1787^{\circ} \mathrm{K}$. The top panel of Figured 5 shows the calculated thermospheric density $\bar{\rho}$ at $500 \mathrm{~km}$, based on the $\mathrm{J} 77$ model and $T_{\infty}(\mathrm{t})$ derived using equation 7. This plot suggests that between the beginning of 13 March to 02:00 UT on 14 March, $\bar{\rho}$ increased by nearly a factor of 6 , from about 1.1 to $6.3 \times 10^{-12} \mathrm{kgm} / \mathrm{cm}^{-3}$. 

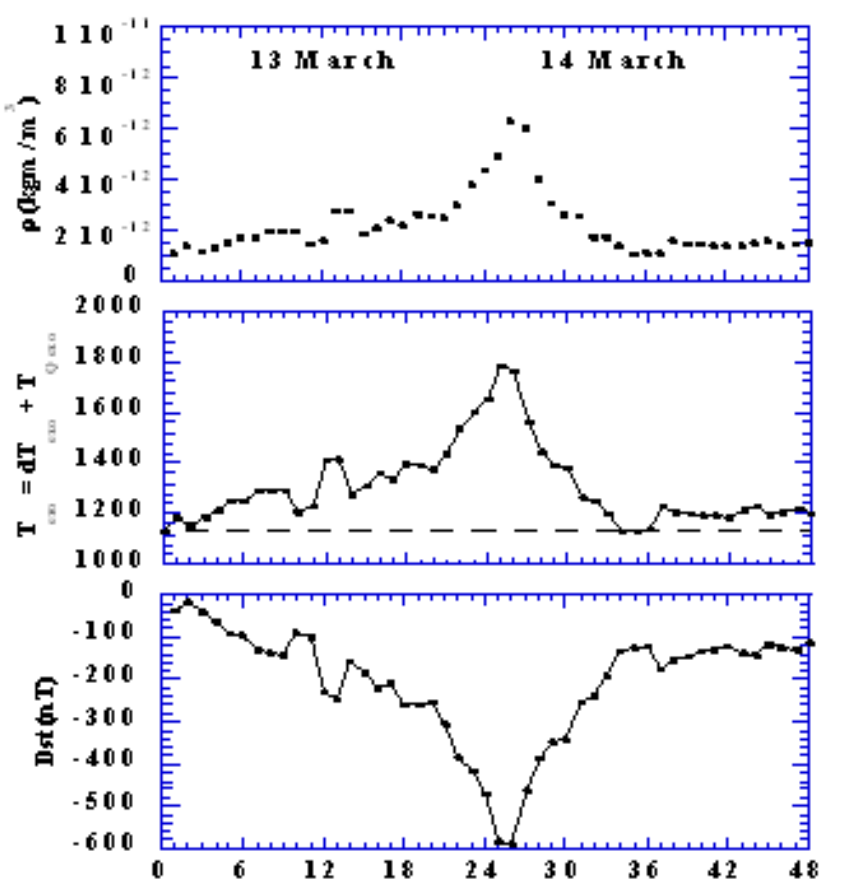

Figure 5. Dst index (bottom), exospheric temperatures (middle) and neutral densities plotted as functions of UT, 13-14 March 1989. The dash line in the middle plot marks $T_{\infty} Q \approx 1135^{\circ} \mathrm{K}$.

\section{Discussion:}

This section has three parts. First, we consider the verisimilitude of our simulation of the global thermosphere during the March 1989 storm by comparing our calculated results with those obtained during comparably large storms of the previous solar cycle when GRACE measurements were available. Second, we comment critically on strengths and weaknesses of the drivendissipative approach to modeling dynamics of the thermosphere during magnetic storms. Third, we reflect on how recent advances in optical surveillance capabilities can be integrated with the driven-dissipative model to improve satellite drag capabilities.

To help understand the space environment during the March 1989 storm, we began by reviewing a method for analyzing GRACE data related to $\bar{\rho}(h), \mathrm{T}_{\infty}$ and $\left\langle\mathrm{F}_{10.7}\right\rangle$. In the cases of both the March 1989 and Halloween 2003 storms, complete sets of interplanetary data in near-Earth space were unavailable. We showed that under such circumstances the Dst index may also be used to predict the temporal evolution of $T_{\infty}$ and $\bar{\rho}(500 \mathrm{~km})$ via equation (7). It is thus useful to compare critical parameters from the March 1989 and Halloween 2003 storms along with two other very large storms that occurred in November 2003 and 2004. These are summarized in Table 1.

Table 1. Parameters used to model the March 1989 and the 3 largest storms of the GRACE era.

\begin{tabular}{|c|c|c|c|c|c|c|}
\hline Date & $\mathrm{Dst}_{\min }(\mathrm{nT})$ & $\left\langle F_{10.7}\right\rangle$ & $T_{Q \infty}\left({ }^{\circ} \mathrm{K}\right)$ & $\delta \mathrm{T}_{\infty \max }\left({ }^{\circ} \mathrm{K}\right)$ & $\alpha_{\mathrm{T}} / \alpha_{D}$ & $\bar{\rho}(500 \mathrm{~km})$ \\
\hline $03 / 13 / 89$ & -589 & 208 & 1135 & 652 & -1.40 & $6.3010^{-12}$ \\
\hline $10 / 30 / 03$ & -401 & 140 & 1056 & 430 & -1.33 & $3.5110^{-12}$ \\
\hline $11 / 20 / 03$ & -467 & 138 & 930 & 621 & -1.33 & $4.1210^{-12}$ \\
\hline $11 / 07 / 04$ & -368 & 106 & 875 & 255 & -1.30 & $1.0610^{-12}$ \\
\hline
\end{tabular}

From left to right, columns in Table 1 lists in chronological order: dates of storm onsets, main-phase Dst minima, $\left\langle\mathrm{F}_{10.7}\right\rangle$ and $T_{\infty \mathrm{Q}}$ on days prior to storm onsets, $\delta T_{\infty \text { max }}$ at the times of Dst ${ }_{\text {min }}$,

coupling coefficient ratios $\left(\boldsymbol{\alpha}_{\mathrm{T}} / \boldsymbol{\alpha}_{\mathrm{D}}\right)$ inferred from contemporary $\left\langle\mathrm{F}_{10.7}\right\rangle$. The last column gives orbit-averaged mass densities (in $\mathrm{kgm} / \mathrm{m}^{3}$ ) at $h \approx 500 \mathrm{~km}$, calculated using inferred or measured $T_{\infty}=T_{\infty \mathrm{Q}}+\delta T_{\infty}$ and $\mathrm{J} 77$. Comparisons of data in columns 3, 4 and 6 confirms that $\left\langle\mathrm{F}_{10.7}\right\rangle$ levels near times large storms onsets play significant roles in the magnitudes of main-phase densities. In nowise does this comment discount the critical forcing role played by the $\varepsilon_{\mathrm{VS}}$ parameter. Almost five decades of satellite measurements in the solar wind provide irrefutable proof that magnetic storm cannot occur, to heat the thermosphere and significantly increase satellite drag, without $\boldsymbol{\varepsilon}_{\mathrm{VS}}$ enhancements. However, consistent with previously reported results by Burke [2011], data in Table 1 confirms a clear dependence on the coupling coefficients on $\alpha_{T}, \alpha_{D}$ and $\left\langle F_{10.7}\right\rangle$. Note that maximum $\bar{\rho}(500 \mathrm{~km})$ was almost a factor of 2 or more higher during the March 1989 than any other of the three recent storms. Given the storm's intensity $\left(D \mathrm{st}_{\min }=-589 \mathrm{nT}\right)$ and unmatched, devastating effects, this result does not appear egregious.

The $\left\langle\mathrm{F}_{10.7}>\right.$ index serves as proxy for the levels of solar EUV fluxes that reach the Earth's upper atmosphere, creating and maintaining its ionization levels. During magnetic disturbances, energy is transferred from the interplanetary medium to the ionosphere-thermosphere system mainly via electromagnetic (Alfvénic) processes. Without an initially high level of ionization being present in the collocated ionosphere thermospheric heating, via ion-neutral collisions, would proceed at much slower rates than observed during actual magnetic storms. This alone suggests that in developing future tracking algorithms and hazardous environment warnings, increased attention be given to both the initial states of the ionosphere as well as to the intensity of interplanetary drivers. Note that Bowman et al., [2008], independently demonstrated that integrating the driven-dissipative model into the USAF satellite drag algorithm reduced stormtime tracking errors by $65 \%$. If nothing else, this indicates the model heads in the right direction. On the other hand, the $35 \%$ persistent error level indicates the presence of as yet unrecognized forces to be included to improve the simulations.

The driven-dissipative model has two free parameters $\left(\alpha_{T}\right.$, and $\tau_{T}$ ) joined with assumptions implicit in the J77 model regarding the spatial distribution of $T_{\infty}$ isotherms. As shown by Burke [2011], errors in values of $\alpha_{T} \tau_{T}$ are easy to identify by discrepancies between measured and predicted $\mathrm{T}_{\infty}$ at the time of a storm's maximum epoch and rectified. The exponential decay time is more problematic. The $\tau_{T}$ value of 6.5 hours used in calculations was based on the measured slopes of $E_{\mathrm{th}}$ traces shown in Figure 2. Higher/lower values of $\tau_{T}$ lead to shorter/longer disturbance periods during which spacecraft are exposed to enhanced drag environments. Implicit in this model was an assumption that the stormtime thermosphere loses excess thermal energy via black body radiation. Wavelengths of maximum intensity would be specified by Wien's Law: $\lambda_{\max } \approx 2900 \mathrm{~mm}$ $K / T_{\infty}$.

Recently, a number of investigators (Lei et al., 2011, 2012; Knipp et al., 2017; Mlynczak et al., 2017) pointed out that regions of significant $\mathrm{NO}$ and $\mathrm{CO}_{2}$ emissions at $\lambda \approx 5.3 \mu \mathrm{m}$ have been observed during large magnetic storms. The intensities of some radiation is such that they are described as "over cooling" events [Knipp et al., 2017]. Mlynczak et al. [2017] stressed the importance of incorporating this information into satellite drag algorithms. The introduction and testing of these photo-chemical considerations into future algorithms describing the stormtime thermosphere seems highly promising. However, its development, contents and predictions lie beyond the scope of the present report.

\section{Acknowledgements:}

The author is grateful to Air Force Research Laboratory colleagues Drs. Frank Marcos and John Wise who introduced him to the thermospheric heating problem and to Dr. Eric Sutton who provided the full set of GRACE measurements between 2002 through the end of 2008. This research was supported in part by AFOSR grant 14-1-FA95500222. 


\section{References}

Bowman, B. R., Tobiska, W. K., Marcos, F. A., Lin, C. S. ,. Huang, C. Y, and Burke W. J.,: 2008, AIAA 2008 6438, AIAA/AAS Astrodynamics Specialist Conference, Honolulu, Hawaii.

Boudouridis, A., Zesta, E., L. R. Lyons, and Anderson, P. S.: 2004, Geophys. Res. Lett., 31, doi:10.1029/2004GL0221252

Bruinsma, S., and Biancale R.: 2003, J. Spacecraft and Rockets, 40, 230.

Bruinsma, S., Tamagnan, D., and R. Biancale 2004, Planet. Space Sci., 52, 297.

Bruinsma, S., Forbes, J. M., Nerem, R. S., and Zhang, X.: 2006, J. Geophys. Res., 111, doi:10. 1029/2005JA011284.

Burke, W. J., Weimer, D. R., and Maynard, N. C.: 1999, J. Geophys. Res., 104, 9989.

Burke, W. J.: 2007, J. Atmos. Solar-Terr. Phys., 69, 279-287.

Burke, W.J., L.C. Gentile, and C.Y. Huang, C. H.: 2007, J. Geophys. Res., 112, A07208, doi:10.1029/2006JA012137.

Burke, W.J., Huang, C. Y., Marcos, F.A, and Wise J. O.: 2007, J. Atmos. Solar-Terr. Phys., 69, 279-287, 2007.

Burke, W. J.: 2008, Mid-Latitude Ionospheric Dynamics and Disturbances, American Geophysical Union Monograph 181, p, 235.

Burke, W. J., Lin, C. S., Hagan, M. P., Huang, C. Y., Weimer, D. R., Wise, J. O., Gentile, L. C., and Marcos F. A., 2009, J. Geophys. Res., 114, doi:10.1029/2008JA013848.

Burke, W. J., 2011, J. Geophys. Res., 116, doi:10.1029/2011JA016437.

Burton, R. K., McPherron, R. L., and Russell, C. T.: 1975, J. Geophys. Res., 80, 4204.

Hill, T. W.: (1984), EOS, Trans. Amer. Geophys. U., 65, 1047.

Jacchia, L. G.: 1970, SAO Special Report 313.

Jacchia, L. G.: 1977, SAO Special Report 375.

Knipp, D. J., Pette, D. V., Kilcommons, L. M., Cruz, A. A., Mlynczak. M. G., Hunt, L. A. and Lin, C.A., 2017, Space Weather, 15, 325-342, doi:10.1002/2016SW001567.

Lei, J., Burns, A. G. Thayer, J. P. Wang, W., Mlynczak, M. G. Hunt, L. A. Dou, X, and Sutton, E.: 2012, J. Geophys Res., 117, doi: 10. 1029/2101JA016.

Mlynczak, M. G., Knipp, D. J., Hunt, L. A., Gaebler, J., Matsuo, T., Kilcommons, L. M., and Young, C. L.: 2017, Space Weather, 16, 363-375, doi: 1002/2017SW001757.

Ober, D. M., Maynard, N. C., and W. J. Burke, W. J.: 2003, J. Geophys. Res., 108, 1467, doi:10.1029/2003JA010154.

Ober, D. M, Wilson, G.R., Maynard, N. C, Burke, W. J., and Siebert, K. D.: 2007, J. Geophys. Res., 112, doi: 10.1029/2006JA012169.

Siscoe, G. L., Erickson, G. M., Sonnerup, B. U. Ö., Maynard, N. C., Schoendorf, J. A, Siebert, K. D., Weimer, D. R.: 2002, J. Geophys. Res., 107(A6), doi: 10.1029/2001JA000109.

Wright, D.: 2007, Physics Today, 60, 35, doi:S-0003-9228-07-10101-7. 